\title{
FRAÇÕES OXIDÁVEIS DO CARBONO ORGÂNICO EM ARGISSOLO VERMELHO-AMARELO SOB SISTEMA DE ALEIAS ${ }^{(1)}$
}

\author{
Arcângelo Loss ${ }^{(2)}$, Marcos Gervasio Pereira ${ }^{(3)}$, Edilene Pereira \\ Ferreira $^{(4)}$, Lauana Lopes dos Santos ${ }^{(4)}$, Sidinei Julio Beutler ${ }^{(4)} \&$ \\ Altamiro Souza de Lima Ferraz Júnior ${ }^{(5)}$
}

\begin{abstract}
RESUMO
O aporte de matéria orgânica ao solo via leguminosas em sistemas de aleias pode ser uma alternativa para o uso sustentável do trópico úmido. O objetivo deste trabalho foi comparar o aporte de matéria orgânica facilmente oxidável proveniente da combinação de resíduos de diferentes leguminosas utilizadas em sistemas de condução de culturas em aleias sob Argissolo Vermelho-Amarelo. Foram avaliadas duas espécies de leguminosas de alta qualidade de resíduos - leucena (Leucaena leucocephala) e guandu (Cajanus cajan), e duas espécies de baixa qualidade de resíduos - sombreiro (Clitoria fairchildiana) e acácia (Acacia mangium), combinadas entre si, nos seguintes tratamentos: sombreiro + guandu; leucena + guandu; acácia + guandu; sombreiro + leucena; leucena + acácia e testemunha sem leguminosa. As amostras de solo foram coletadas nas profundidades de 0-5 e 5$10 \mathrm{~cm}$, nas entrelinhas. Foi quantificado e fracionado o $\mathrm{C}$ orgânico total (COT), estratificado em quatro frações (F1, F2, F3 e F4) com graus decrescentes de oxidação. As áreas com sombreiro/leucena, acácia/leucena e acácia/guandu apresentaram os maiores teores de COT (0-5 cm), e a área testemunha, os menores $(5-10 \mathrm{~cm})$. A maior proporção do COT estava nas frações F1+F2; a área testemunha teve os menores teores de $\mathrm{C}$ nessas frações. $\mathrm{O}$ tratamento acácia/guandu apresentou maiores teores de $\mathrm{C}$ na fração $\mathrm{F} 1$ nas duas profundidades. $\mathrm{O}$ tratamento leucena/ guandu apresentou as maiores proporções do COT nas frações F3 + F4. Os resultados indicam que, no cultivo em sistema de aleias, a combinação de leguminosas de alta e baixa qualidade de resíduos (acácia/guandu) favorece o aumento de matéria orgânica facilmente decomponível, enquanto o uso de
\end{abstract}

\footnotetext{
(1) Recebido para publicação em outubro de 2008 e aprovado em maio de 2009.

(2) Doutorando do Curso de Pós-Graduação em Agronomia-Ciência do Solo, Universidade Federal Rural do Rio de Janeiro UFRRJ. BR 465, Km 7, CEP 23890-000 Seropédica (RJ). Bolsista CNPq. E-mail: arcangeloloss@yahoo.com.br

(3) Professor Associado II do Departamento de Solos, Instituto de Agronomia, UFRRJ. Bolsista CNPq. E-mail: gervasio@ufrrj.br

(4) Discente do Curso de Agronomia, UFRRJ. Bolsista CNPq/PIBIC. E-mails: edilenepereiraferreira@hotmail.com; lauanalsantos@hotmail.com; s.parana@yahoo.com.br

(5) Professor Adjunto IV, Universidade Estadual do Maranhão - UEMA. Cidade Universitária Paulo VI, Tirirical, CEP 65065-970 São Luis (MA). E-mail: aferrazjr@yahoo.com.br
} 
leguminosas de alta qualidade de resíduos favorece maiores proporções de $\mathrm{C}$ somente nas frações mais resistentes (F3 + F4).

Termos de indexação: carbono lábil, leguminosas, qualidade de resíduos e agrossistema.

\title{
SUMMARY: OXIDIZABLE ORGANIC CARBON FRACTIONS OF AN ULTISOL UNDER AN ALLEY CROPPING SYSTEM
}

\begin{abstract}
The input of organic matter to the soil by legumes in alley cropping systems may be an alternative for a sustainable soil use in the humid tropics. The objective of this study was to compare the input of oxidizable organic matter in a combination of residues from different legumes in alley cropping systems on an Ultisol. Two legume species were evaluated: leucena (Leucaena leucocephala) and pigeon pea (Cajanus cajan), which have high-quality residues, and Philippine pigeonwings (Clitoria fairchildiana) and acacia (Acacia mangium), with low-quality residues. The residues were combined in the following treatments: Philippine pigeonwings + pigeon pea; leucena + pigeon pea; acacia + pigeon pea; Philippine pigeonwings + leucena; leucena + acacia; an area without legumes was used as control. Soil samples were taken from the layers $0-5$ and 5-10 cm in the crop inter-row. Total organic carbon (TOC) was quantified and divided into four fractions (F1, F2, F3 and F4) with decreasing oxidation degrees. In the areas with Philippine pigeonwings/leucena, acacia/leucena and acacia/pigeon pea the TOC content was higher at the depth of $0-5 \mathrm{~cm}$. In the area without legumes the TOC content was the lowest at the depth of 5-10 cm. The highest TOC proportion was observed in the $F 1+F 2$ fractions, and the carbon contents for these fractions were lower in the area without legumes. In the acacia/pigeon pea treatment the C content was higher in the F1 fraction at both depths, while the TOC proportions were greater in the treatment leucaena/pigeon pea in the fractions F3 + F4. The results indicate that the combination of high and low quality residues (acacia / pigeon pea) favors the increase of easily decomposable organic matter, while the use of high-quality residues resulted in higher carbon proportions in the more resistant fractions $(F 3+F 4)$.
\end{abstract}

Index terms: labile carbon, legumes, residue quality, agrosystem.

\section{INTRODUÇÃO}

Nas últimas décadas, novos conceitos de sistemas de produção agrícola, baseados na conservação do solo, na diversificação de culturas, na reciclagem de nutrientes, no uso sistemático de adubos orgânicos e em outras práticas alternativas, têm sido desenvolvidos na tentativa de equilibrar a produtividade com a conservação do meio ambiente. Uma das modalidades de produção que atende a esse conceito é o sistema de cultivo em aleias (Salmi et al., 2006).

O cultivo em aleias (alley crops) é um sistema agroflorestal simultâneo que consiste na associação de árvores e, ou, arbustos, geralmente fixadores de $\mathrm{N}_{2}$, intercalados em faixas com culturas anuais. As árvores ou arbustos são podados periodicamente para utilização dos caules e das folhas como adubo verde e, ou, lenha, para promover melhorias na fertilidade do solo, e, ou, ser utilizados como forragem de alta qualidade. Este sistema foi inspirado nas práticas dirigidas à recuperação de áreas em pousio melhorado mediante o uso de adubos verdes (Mac Dicken \& Vergara, 1990; Kang et al., 1997).
Em tal contexto, o adequado manejo da matéria orgânica do solo (MOS) é crítico para manter os aspectos econômicos e ambientais da produção de alimentos, especialmente em zonas áridas e semiáridas. Alguns estudos mostram que práticas agroecológicas, como adubação verde com leguminosas, são importantes para melhorar a qualidade e a quantidade de matéria orgânica (Breman \& Kessler, 1997; Nair et al., 1999; Sharrow \& Ismail, 2004; Loss et al., 2009). Além disso, em regiões semiáridas, sistemas florestais e, ou, cultivo em aleias podem ser uma forma eficiente de aumentar os teores deMOS.

O cultivo em aleias baseia-se no princípio de que é possível obter produção e sustentabilidade na terra, com diversificação de fontes de renda para o pequeno produtor, quando os métodos de conservação e reabilitação são introduzidos antes que ocorra degradação dos recursos (Kaya \& Nair, 2001). A adição de matéria orgânica na forma de material podado das árvores pode alterar significativamente as condições físicas e químicas do solo no curto prazo, que não seriam alcançadas somente com fertilizantes químicos (Aihou et al., 1999; Aguiar, 2006). 
Características como melhoria da qualidade do solo e aumento da produtividade das culturas alimentares e dos rendimentos das propriedades rurais têm sido atribuídas ao sistema de cultivo em aleias, mas o sucesso de um sistema deste tipo está relacionado com a quantidade e qualidade do material podado das árvores, com a quantidade de nutrientes liberados dos resíduos durante o processo de decomposição e com o tempo de liberação de nutrientes para satisfazer as necessidades das culturas subsequentes (Mendonça $\&$ Stott, 2003).

A qualidade dos resíduos vegetais está relacionada principalmente com a relação $\mathrm{C} / \mathrm{N}$ e a disponibilidade de $\mathrm{N}$ mineral na solução do solo, influenciando diretamente na taxa de decomposição dos resíduos vegetais (Ceretta et al., 2002)

As leguminosas com raízes profundas podem potencialmente interceptar nutrientes percolados ao longo do perfil do solo e acessar aqueles acumulados nas camadas abaixo da zona radicular de culturas anuais. Esses nutrientes absorvidos pelo sistema radicular das árvores tornam-se insumos quando transferidos para a superfície do solo na forma de serrapilheira e de outros resíduos vegetais (Aguiar, 2006). A quantidade de $\mathrm{C}$ e $\mathrm{N}$ ciclados por meio da decomposição de raízes finas pode ser igual ou maior do que a de material podado ciclado deixado na superfície do solo (José et al., 2000).

Com o objetivo de avaliar diferentes frações de oxidação do C do solo, Chan et al. (2001) introduziram uma modificação no método clássico de determinação do C desenvolvido por Walkley \& Black (1934). No método original, o $\mathrm{C}$ era determinado por uma única concentração de ácido sulfúrico $\left(12 \mathrm{~mol} \mathrm{~L}^{-1}\right)$. Com a modificação proposta por Chan et al. (2001), foi possível separar quatro frações com graus decrescentes de oxidação, por meio da utilização de concentrações crescentes de ácido sulfúrico. Foram denominadas de F1, F2, F3 e F4, correspondendo, respectivamente, às concentrações de 3, 6, 9 e $12 \mathrm{~mol} \mathrm{~L}^{-1}$ de ácido sulfúrico.

As frações F1 e F2 estão associadas à disponibilidade de nutrientes e à formação de macroagregados (Blair et al., 1995; Chan et al., 2001), sendo a fração F1 a de maior labilidade no solo e altamente correlacionada com a fração leve livre da MOS (Maia et al., 2007). Já as frações F3 e F4 estão relacionadas a compostos de maior estabilidade química e massa molar, oriundos da decomposição e humificação da MOS (Stevenson, 1994). A fração F4 é a mais resistente do solo, sendo denominada "compartimento passivo" nos modelos de simulação da MOS, com tempo de reciclagem de até 2.000 anos (Chan et al., 2001).

O objetivo deste trabalho foi comparar o aporte de matéria orgânica facilmente oxidável proveniente da combinação de resíduos de diferentes leguminosas utilizadas em sistemas de condução de culturas em aleias sob Argissolo Vermelho-Amarelo.

\section{MATERIAL E MÉTODOS}

O experimento foi instalado na área experimental da Universidade Estadual do Maranhão, São Luís $\left(2^{\circ}\right.$ 30 ' S, $44^{\circ} 18$ ' W), em janeiro de 2002 , no início do período chuvoso. O clima da região, segundo Köppen, é do tipo Aw', equatorial quente e úmido, com duas estações bem definidas: uma estação chuvosa, que se estende de janeiro a junho, e uma estação seca, com déficit hídrico acentuado de julho a dezembro. As precipitações pluviais variam de 1.700 a $2.300 \mathrm{~mm}$ anuais, e mais de $80 \%$ ocorrem de janeiro a maio. A temperatura média situa-se em torno de $26,7^{\circ} \mathrm{C}$, e as máximas variam de 28 a $37^{\circ} \mathrm{C}$ e as mínimas entre 20 e $23^{\circ} \mathrm{C}$ (Aguiar, 2006).

O solo da área experimental foi classificado como Argissolo Vermelho-Amarelo distrófico arênico, textura franco-arenosa no horizonte A (Embrapa, 2006). A caracterização química e física do solo feita em 2002 , na profundidade de $0-20 \mathrm{~cm}$, encontra-se no quadro 1.

Com base na análise de solo de 2002 (Quadro 1), a calagem do solo foi feita em 2003, com aplicação superficial de cal hidratada, na dosagem de $1 \mathrm{Mg} \mathrm{ha}^{-1}$, com $30 \%$ de $\mathrm{CaO}$ e $22 \%$ de $\mathrm{MgO}$, o que equivale a $214 \mathrm{~kg}$ de Ca e $132 \mathrm{~kg}$ de $\mathrm{Mg}$.

Foram avaliadas duas espécies de leguminosas de alta qualidade de resíduos - leucena (Leucaena

Quadro 1. Características químicas e físicas do Argissolo Vermelho-Amarelo antes da implantação do sistema de aleias

\begin{tabular}{|c|c|c|c|c|c|c|c|c|c|c|c|c|}
\hline \multicolumn{13}{|c|}{ Característica física } \\
\hline \multirow[t]{2}{*}{$\mathrm{Ds}^{(1)}$} & \multicolumn{3}{|c|}{ Granulometria } & \multirow[t]{2}{*}{ Textura } & \multicolumn{8}{|c|}{ Característica química } \\
\hline & Areia & Silte & Argila & & $\overline{\mathrm{pH} \mathrm{CaCl}}{ }_{2}$ & $\mathrm{Ca}^{2+}$ & $\mathrm{Mg}^{2+}$ & $\mathbf{K}$ & $\mathrm{Al}^{3+}$ & $\mathrm{H}+\mathrm{Al}$ & $\mathbf{P}$ & $\overline{\mathrm{CO}}$ \\
\hline $\mathrm{Mg} \mathrm{m}^{-3}$ & 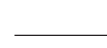 & $\mathrm{g} \mathrm{kg}^{-1}$ & 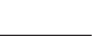 & & & 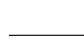 & - & $\mathrm{mol}_{\mathrm{c}} \mathrm{k}$ & 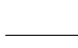 & ـ & $\mathrm{mg} \mathrm{kg}^{-1}$ & $\mathrm{~g} \mathrm{~kg}^{-1}$ \\
\hline 1,69 & 780 & 120 & 100 & Franco arenosa & 4,3 & 0,2 & 0,3 & 0,12 & 0,3 & 3,3 & $<1$ & 7,54 \\
\hline
\end{tabular}


leucocephala) e guandu (Cajanus cajan), e duas espécies de baixa qualidade de resíduos - sombreiro (Clitoria fairchildiana) e acácia (Acacia mangium), combinadas entre si, nos seguintes tratamentos: sombreiro + guandu; leucena + guandu; acácia + guandu; sombreiro + leucena; leucena + acácia e uma área testemunha, sem leguminosa. A definição de resíduos de alta e baixa qualidade foi feita segundo Young (1997), que define os resíduos de alta qualidade como aqueles que apresentam alto teor de $\mathrm{N}$, baixa quantidade de lignina e polifenóis; e o inverso deve ser denominado de resíduo de baixa qualidade. A caracterização química e a matéria seca das leguminosas utilizadas no experimento são apresentadas no quadro 2 .

As sementes de leucena e sombreiro foram coletadas de espécies locais. Para a semeadura do guandu, foi utilizado o cultivar taifeiro. Para o plantio da acácia, foram utilizadas mudas de dois meses de idade, produzidas localmente (Aguiar, 2006). Utilizouse o delineamento experimental em blocos ao acaso, com seis tratamentos e quatro repetições. As leguminosas foram dispostas em linhas simples, espaçadas de 0,5 m entre plantas e 4,0 m entre linhas, em parcelas de $21,0 \times 4,0 \mathrm{~m}$.

Em cada parcela, foram plantados milho (Zea mays L.) e arroz (Oriza sativa L.) sobre os resíduos vegetais das leguminosas, sem preparo do solo. O milho, cultivar AG 1051, foi semeado com matraca em janeiro de cada ano, com densidade de cinco sementes por metro, em sulcos espaçados com $1 \mathrm{~m}$ entre linhas. Já o arroz de terras altas, cultivar Bonança, foi semeado manualmente no mesmo período que o milho, com 100 sementes por metro, com espaçamento de $0,40 \mathrm{~m}$ entre linhas.

As podas das leguminosas foram realizadas em janeiro de 2004, 2005 e 2006, após a germinação do milho e arroz, à altura de aproximadamente $50 \mathrm{~cm}$. O corte não foi realizado em 2003 devido ao difícil estabelecimento das leguminosas, principalmente da leucena, por causa da baixa fertilidade natural do solo (Quadro 2). No ano de 2006, a leucena foi submetida a um segundo corte 35 dias após o plantio, para evitar o sombreamento. As quantidades de biomassa aplicadas ao solo, em kg parcela ${ }^{-1}$ de matéria fresca, foram igualmente distribuídas entre todas as parcelas de cada tratamento, mantendo-se os resíduos na superfície do solo.

A coleta das amostras de solo foi realizada em dezembro de 2007. Para cada tratamento, foram coletadas quatro amostras compostas por três amostras simples nas profundidades de $0-5$ e $5-10 \mathrm{~cm}$, nas entrelinhas. Foram quantificados os teores de $\mathrm{C}$ orgânico total (COT) do solo, segundo Yeomans \& Bremner (1988), e realizado o fracionamento do $\mathrm{C}$ por graus de oxidação (Chan et al., 2001).

Amostras de 0,5 g de solo foram acondicionadas em erlenmeyer de $250 \mathrm{~mL}$, onde se adicionaram $10 \mathrm{~mL} \mathrm{~K}_{2} \mathrm{Cr}_{2} \mathrm{O}_{7} 0,167 \mathrm{~mol} \mathrm{~L}^{-1}$ e quantidades de $\mathrm{H}_{2} \mathrm{SO}_{4}$ correspondentes às concentrações de $3,6,9$ e $12 \mathrm{~mol} \mathrm{~L}^{-1}$. A oxidação foi realizada sem fonte externa de calor e a titulação dos extratos foi feita com uma solução de $\mathrm{Fe}\left(\mathrm{NH}_{4}\right)_{2}\left(\mathrm{SO}_{4}\right)_{2} \cdot 6 \mathrm{H}_{2} \mathrm{O} 0,5 \mathrm{~mol} \mathrm{~L}^{-1}$, utilizando-se como indicador a fenantrolina. Foram obtidas quatro frações, com graus decrescentes de oxidação do $\mathrm{C}$ :

- Fração 1(F1): C oxidado por $\mathrm{K}_{2} \mathrm{Cr}_{2} \mathrm{O}_{7}$ em meio ácido de $3 \mathrm{~mol} \mathrm{~L}^{-1}$ de $\mathrm{H}_{2} \mathrm{SO}_{4}$.

- Fração 2 (F2): diferença do C oxidado por $\mathrm{K}_{2} \mathrm{Cr}_{2} \mathrm{O}_{7}$ em meio ácido com 6 e $3 \mathrm{~mol} \mathrm{~L}^{-1} \mathrm{de}_{2} \mathrm{SO}_{4}$.

- Fração 3 (F3): diferença do C oxidado por $\mathrm{K}_{2} \mathrm{Cr}_{2} \mathrm{O}_{7}$ em meio ácido com 9 e $6 \mathrm{~mol} \mathrm{~L}^{-1}$ de $\mathrm{H}_{2} \mathrm{SO}_{4}$.

- Fração 4 (F4): diferença do C oxidado por $\mathrm{K}_{2} \mathrm{Cr}_{2} \mathrm{O}_{7}$ em meio ácido com 12 e $9 \mathrm{~mol} \mathrm{~L}^{-1} \mathrm{de}_{2} \mathrm{SO}_{4}$.

Os resultados foram submetidos à aplicação do teste de normalidade (teste de Lilliefors), à avaliação da homogeneidade da variância (teste de Cochran e Barttlet) e à análise de variância com aplicação do teste F. Não foi necessária a transformação de nenhum dado. Os valores médios, quando significativos pelo teste $\mathrm{F}$, foram comparados entre si pelo teste de Scott-Knott a $5 \%$.

Quadro 2. Caracterização química das leguminosas feita em folhas e galhos tenros, em janeiro de 2005, e matéria seca produzida pelas leguminosas nos três anos de corte

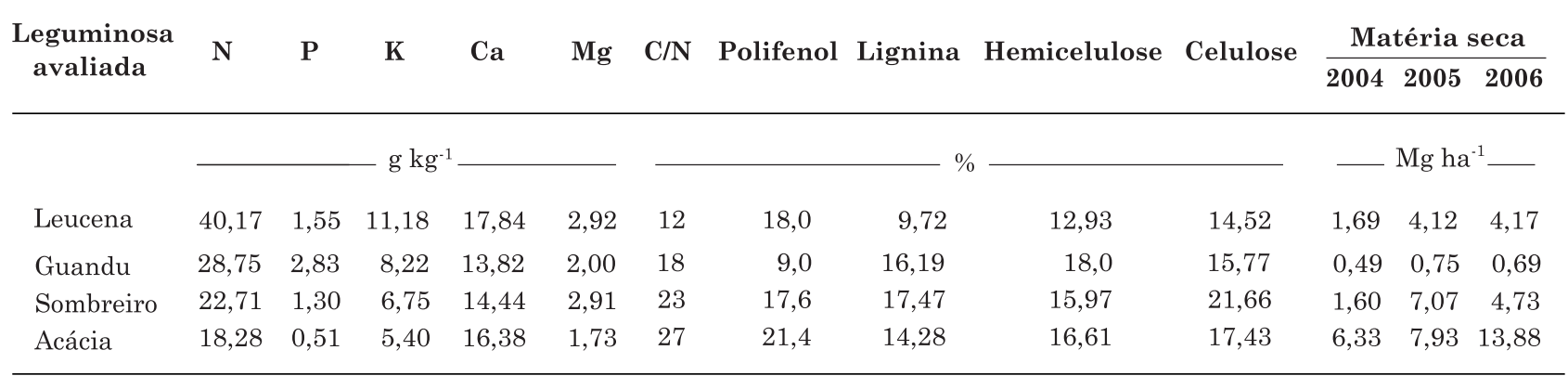

Dados extraídos de Aguiar (2006). 


\section{RESULTADOS E DISCUSSÃO}

As áreas com sombreiro/leucena, acácia/leucena e acácia/guandu apresentaram os maiores teores de COT na profundidade de $0-5 \mathrm{~cm}$ (Quadro 3), sendo esses teores todos superiores aos encontrados no início do experimento (Quadro 2). Os maiores teores de COT podem ser decorrentes da maior quantidade de resíduos vegetais produzidos pelas espécies de leguminosas avaliadas, destacando-se a acácia e o guandu com as maiores e menores produtividades de matéria seca, respectivamente, nos três anos avaliados (Quadro 2).

$\mathrm{Na}$ profundidade de $5-10 \mathrm{~cm}$, verificou-se que apenas a área testemunha diferiu das demais, apresentando os menores valores de COT. Esse resultado demonstra a importância do sistema de cultivo em aleias para a manutenção e, ou, melhoria dos teores de COT em áreas de baixa fertilidade natural e solos com textura arenosa na região do trópico úmido.

Observou-se que as parcelas com sombreiro/leucena e acácia/guandu apresentaram os maiores valores da fração F1, sendo também verificados os maiores teores de COT nessas áreas (Quadro 3). Pode-se destacar que, no tratamento acácia/guandu, ocorreram os maiores teores de $\mathrm{C}$ na fração $\mathrm{F} 1$ nas duas profundidades. Este padrão pode ser decorrente da maior relação $\mathrm{C} / \mathrm{N}$ encontrada na acácia quando comparada às demais leguminosas Quadro 2), favorecendo a deposição de resíduos culturais de degradação mais lenta e, portanto, propiciando o acúmulo de $\mathrm{C}$ devido a sua maior relação $\mathrm{C} / \mathrm{N}$.
O sistema de cultivo em aleias para o tratamento acácia/guandu está propiciando aumentos nos teores de $\mathrm{C}$ da fração $\mathrm{F} 1$, quando comparado aos demais tratamentos, principalmente em relação à área testemunha. Os sistemas de manejo que adicionam matéria orgânica ao solo via resíduos vegetais promovem aumento dessa fração (Blair et al., 1995; Chan et al., 2001; Rangel et al., 2008; Beutler et al., 2008; Moraes et al., 2008), sendo este aumento relacionado principalmente com a fração leve livre da MOS (Maia et al., 2007).

Avaliando o C orgânico em um Luvissolo textura arenosa, sob agrossilvicultura e sistemas de agricultura convencionais na região semiárida de Ceará, Maia et al. (2007) encontraram resultados semelhantes ao deste estudo. Os autores observaram maiores teores de $\mathrm{C}$ na fração $\mathrm{F} 1$ na camada superficial do solo, associando sua maior concentração à maior disponibilidade de resíduos vegetais (fração leve livre) provenientes dos sistemas avaliados.

De maneira geral, a maior proporção do COT ocorreu nas frações F1+F2, destacando-se a profundidade de $0-5 \mathrm{~cm}$ com os maiores valores dessas proporções. A área testemunha apresentou comportamento divergente, sendo as maiores proporções de COT observadas nas frações F3 + F4. Os menores teores de $\mathrm{C}$ das frações $\mathrm{F} 1$ e $\mathrm{F} 2$ foram encontrados nesta área (Quadro 3).

Esses resultados indicam que nos solos das áreas cultivadas em sistema de aleias há predominância no

Quadro 3. Frações oxidáveis de carbono de um Argissolo Vermelho-Amarelo sob sistema de cultivo em aleias

\begin{tabular}{|c|c|c|c|c|c|}
\hline \multirow{2}{*}{ Tratamento } & \multicolumn{5}{|c|}{ Frações oxidáveis de carbono } \\
\hline & F1 & F2 & F3 & F4 & COT \\
\hline & \multicolumn{5}{|c|}{$\mathrm{g} \mathrm{kg}^{-1}$} \\
\hline & & & $0-5 \mathrm{~cm}$ & & \\
\hline Sombreiro/leucena & $4,95(30) \mathrm{A}$ & $2,70(16) \mathrm{A}$ & $2,10(13) \mathrm{C}$ & $4,12(25) \mathrm{A}$ & $16,62 \mathrm{~A}$ \\
\hline Acácia/leucena & $3,15(19) \mathrm{C}$ & $3,38(21) \mathrm{A}$ & $3,82(23) \mathrm{A}$ & $3,00(18) \mathrm{B}$ & $16,28 \mathrm{~A}$ \\
\hline Leucena/guandu & $4,12(29) \mathrm{B}$ & $3,60(25) \mathrm{A}$ & $2,85(20) \mathrm{B}$ & $3,60(25) \mathrm{A}$ & $14,14 \mathrm{~B}$ \\
\hline Acácia/guandu & $5,25(34) \mathrm{A}$ & $3,52(23) \mathrm{A}$ & $2,62(17) \mathrm{B}$ & $2,78(18) \mathrm{B}$ & $15,24 \mathrm{~A}$ \\
\hline Sombreiro/guandu & $3,45(26) \mathrm{C}$ & $3,22(24) \mathrm{A}$ & $1,50(11) \mathrm{C}$ & $3,68(28) \mathrm{A}$ & $13,22 \mathrm{~B}$ \\
\hline Testemunha & $1,95(17) \mathrm{D}$ & $1,58(14) \mathrm{B}$ & $2,17(19) \mathrm{C}$ & $4,42(39) \mathrm{A}$ & $11,35 \mathrm{~B}$ \\
\hline Fcal & $30,33 * *$ & $11,56^{* *}$ & $11,14^{* *}$ & $6,23 * *$ & $5,05^{* *}$ \\
\hline CV (\%) & 11,66 & 15,05 & $\begin{array}{l}18,95 \\
5-10 \mathrm{~cm}\end{array}$ & 14,05 & 12,26 \\
\hline Sombreiro/leucena & $2,32(21) \mathrm{C}$ & $2,48(23) \mathrm{C}$ & $1,72(15) \mathrm{B}$ & $3,60(33) \mathrm{A}$ & $10,95 \mathrm{~A}$ \\
\hline Acácia/leucena & $2,32(20) \mathrm{C}$ & $3,75(32) \mathrm{A}$ & $1,35(11) \mathrm{B}$ & $3,15(27) \mathrm{A}$ & $11,73 \mathrm{~A}$ \\
\hline Leucena/guandu & $3,30(32) \mathrm{B}$ & $2,10(20) \mathrm{C}$ & $2,48(24) \mathrm{A}$ & $2,78(27) \mathrm{B}$ & $10,44 \mathrm{~A}$ \\
\hline Acácia/guandu & $3,98(37) \mathrm{A}$ & $1,95(18) \mathrm{C}$ & $1,64(15) \mathrm{B}$ & $2,10(20) \mathrm{B}$ & $10,71 \mathrm{~A}$ \\
\hline Sombreiro/guandu & $1,73(17) \mathrm{D}$ & $2,78(27) \mathrm{B}$ & $1,65(16) \mathrm{B}$ & $3,22(31) \mathrm{A}$ & $10,31 \mathrm{~A}$ \\
\hline Testemunha & $1,58(31) \mathrm{D}$ & $1,28(15) \mathrm{D}$ & $1,35(15) \mathrm{B}$ & $2,32(27) \mathrm{B}$ & $8,72 \mathrm{~B}$ \\
\hline Fcal & $23,41^{* *}$ & $12,66^{* *}$ & $4,70 * *$ & $5,62^{* *}$ & $3,71^{*}$ \\
\hline CV (\%) & 15,14 & 19,77 & 22,40 & 16,85 & 9,88 \\
\hline
\end{tabular}

Letras iguais na coluna não diferem entre si (Skott-Knott <0,05). *significativo a $5 \% \mathrm{e}$ ** significativo a $1 \%$ pelo teste $\mathrm{F}$. Valores entre parênteses representam a relação de cada fração (F1, F2, F3 e F4) com o COT. Fcal: Fcalculado; CV: coeficiente de variação. 
solo de matéria orgânica de maior biodisponibilidade, devido ao aporte de resíduos vegetais provenientes das leguminosas utilizadas. Avaliando as frações oxidáveis de C orgânico de Argissolo Vermelho-Amarelo textura franco-arenosa no horizonte A, cultivado com frutíferas e oleráceas sob manejo orgânico em Seropédica, RJ, Beutler et al. (2008) também encontraram maiores proporções do COT nas frações F1 e F2. Os autores atribuíram estas maiores proporções principalmente ao aporte e decomposição dos resíduos vegetais adicionados ao solo.

Resultados similares ao deste estudo também foram encontrados por Freitas et al. (2004), avaliando a MOS em sistemas de adubação orgânica e mineral. Os autores verificaram que mais da metade do COT ocorria nas frações mais lábeis (F1 e F2) independente do tipo de adubação empregada. Esses resultados suportam a análise de Chan et al. (2001), que sugerem que a determinação do COT seja feita com menores concentrações de $\mathrm{H}_{2} \mathrm{SO}_{4}$ do que a recomendada por Walkley \& Black (1934) para monitoramento do uso do solo (Freitas et al., 2004), como sistemas de manejo que promovam adição de MOS para melhoria de suas propriedades químicas e físicas.

Na profundidade de $0-5 \mathrm{~cm}$, a soma das frações F3 + F4 das leguminosas representou menores percentagens referente ao COT quando comparadas à testemunha, sendo verificado $58 \%$ das frações F3 + F4 referente ao COT (Quadro 3). Este padrão indica que nas áreas onde há cultivo de leguminosas em aleias, o aporte da palhada está acarretando maiores teores de COT nas frações F1 e F2.

Avaliando as frações de $\mathrm{C}$ oxidáveis em sistema de cultivo com arroz e milho por 19 anos na Índia, com adubação verde e uso de palhada, Manjuder et al. (2008) verificaram que as frações F3 e F4 representaram apenas $39 \%$ do COT, sendo a maior parte do COT encontrado nas frações F1 e F2. Os autores concluíram que a fração $\mathrm{F} 1$ pode ser considerada um bom indicador de sustentabilidade do sistema, uma vez que foram altamente correlacionadas com a produtividade das culturas de milho e arroz.

A combinação da leguminosa guandu, de alta qualidade de resíduos, com a leguminosa acácia, de baixa qualidade, está promovendo maiores teores de COT $(0-5 \mathrm{~cm})$ e de C na fração $\mathrm{F} 1(0-5$ e $5-10 \mathrm{~cm})$, quando comparada à combinação das duas leguminosas com alta qualidade de resíduos, leucena/guandu (Quadro 3).

Este comportamento demonstra que as culturas plantadas no sistema de aleias com acácia/guandu serão beneficiadas indiretamente com a matéria orgânica de maior labilidade, que propiciará melhoria nas propriedades físicas e químicas do solo, acarretando melhor crescimento radicular das culturas comerciais e, posteriormente, aumento na absorção dos nutrientes. Estes resultados são corroborados pelos encontrados por Manjuder et al. (2008).
Em um sistema integrado de produção seria interessante ter um balanço de $\mathrm{C}$ nas frações oxidáveis, com as mesmas proporções de $\mathrm{C}$ entre as frações, sendo parte de matéria orgânica facilmente decomponível para mineralização dos nutrientes e outra parte mais resistente no solo para melhoria e, ou, manutenção das propriedades físicas do solo. Entretanto, na região do trópico úmido, em ambiente de solo de textura arenosa, clima equatorial quente e úmido e estação chuvosa, que se estende de janeiro a junho, com altas taxas de insolação, devido à maior proximidade com a linha do Equador, seria desejável um aumento do teor de $\mathrm{C}$ das frações oxidáveis mais resistentes (F3 e F4) para liberação mais lenta de nutrientes associada à melhoria das características físicas do solo.

Dentre as leguminosas avaliadas, destaca-se aquela que promoveu maior proporção de $\mathrm{C}$ nas frações F3 + F4, nas duas profundidades, a combinação leucena/guandu, ambas de alta qualidade de resíduos. $\mathrm{Na}$ profundidade de $0-5 \mathrm{~cm}$, essas leguminosas representaram $45 \%$ do COT para F3 + F4, e, na profundidade de $5-10 \mathrm{~cm}$, verificou-se que $51 \%$ do COT estava nessas frações (Quadro 3). Esses maiores teores de $\mathrm{C}$ são decorrentes da menor relação $\mathrm{C} / \mathrm{N}$ dessas leguminosas (Quadro 2), o que as caracterizam como leguminosas de alta qualidade de resíduos, apresentando menor taxa de decomposição dos resíduos vegetais quando comparadas com as leguminosas de baixa qualidade de resíduos.

Também podem-se destacar como boas combinações de leguminosas, tanto para favorecer frações mais lábeis do $\mathrm{C}$ (F1 e F2) como também as frações mais resistentes (F3 e F4), o uso do sombreiro, sendo sombreiro/leucena e sombreiro/guandu e as associações que apresentaram 48 e $47 \%$, respectivamente, de proporção do COT nas frações F3 + F4, para a profundidade de 5-10 cm (Quadro 3).

Segundo Moura \& Aguiar (2007), o uso de leguminosas arbóreas nativas, como o sombreiro, por sua perfeita adaptabilidade e grande capacidade de produção de resíduos de baixa qualidade e grande durabilidade na superficie do solo, aumenta a demanda provisória por N no sistema, que pode ser atendida pelos resíduos de leguminosas anuais semeadas no final do período chuvoso, junto à cultura de safrinha.

\section{CONCLUSÕES}

1. O sistema de cultivo em aleias aumentou os teores de COT, quando comparado à área testemunha, na profundidade de $5-10 \mathrm{~cm}$.

2. O tratamento acácia/guandu favoreceu maiores aportes de matéria orgânica facilmente decomponível (fração F1), enquanto o tratamento leucena/guandu propiciou maiores aportes de matéria orgânica mais resistente (fração F3 + F4). 
3. O cultivo em aleias, em solo arenoso e de baixa fertilidade natural, proporcionou maior disponibilidade de matéria orgânica nas frações mais lábeis quando comparada à testemunha, sendo uma alternativa para o uso sustentável do solo do trópico úmido.

\section{AGRADECIMENTOS}

À Universidade Estadual do Maranhão (UEMA) e à Universidade Federal Rural do Rio de Janeiro (UFRRJ).

\section{LITERATURA CITADA}

AGUIAR, A.C.F. Sustentabilidade do sistema plantio direto em Argissolo no trópico úmido. Botucatu, Universidade Estadual Paulista, 2006. 55p. (Tese de Doutorado)

AIHOU, K.; SANGINGA, N.; VANLAUWE, B.; LYASSE, O.; DIELS, J. \& MERCKX, R. Alley cropping in the moist savanna of West-Africa: I. Restoration and maintenance of soil fertility on "terre de barre" in Bénin Republic. Agrofor. Syst., 42:213-227, 1999.

BEUTLER, S.J.; LOSS, A.; PEREIRA, M.G.; ANJOS, L.H.C.; FERREIRA, E.P.; SANTOS, L.L. \& SILVA, E.M.R. Frações oxidáveis do carbono orgânico cultivado sob manejo orgânico. In: FERTBIO, 2008. Desafios para o uso do solo com eficiência e qualidade ambiental. Londrina, 2008. Anais. Londrina, 2008. CD ROM.

BLAIR, G.J.; LEFROY, R.D.B. \& LISLE, L. Soil carbon fractions based on their degree of oxidation, and the development of a carbon management index for agricultural systems. Austr. J. Agric. Res., 46:1459-1466, 1995.

BREMAN, H. \& KESSLER, J.J. The potential benefits of agroforetry in the Sahel and other semi-arid regions. Eur J. Agron, 7:25-33, 1997.

CERETTA, C.A.; BASSO, C.J.; HERBES, M.G.; POLETTO, N. \& SILVEIRA, M.J. Produção e decomposição de fitomassa de plantas invernais de cobertura de solo e milho, sob diferentes manejos da adubação nitrogenada. Ci. Rural, $32: 49-54,2002$.

CHAN, K.Y.; BOWMAN, A. \& OATES, A. Oxidizidable organic carbon fractions and soil quality changes in an Oxic Paleustalf under different pasture ley. Soil Sci., 166:6167, 2001.

EMPRESA BRASILEIRA DE PESQUISA AGROPECUÁRIA EMBRAPA. Sistema brasileiro de classificação de solos. 2.ed. Brasília, Embrapa Produção de informação; Rio de Janeiro, Embrapa Solos, 2006. 312p.

FREITAS, A.G.; MATOS, E.S. \& MENDONÇA, E.S. Matéria orgânica e estabilidade de agregados em diferentes sistemas de adubação. In: FERTBIO, Lages, 2004. Anais. Lages, UDESC/SBCS, 2004. CD-ROM.
JOSE, S.; GILLESPIE, A.R.; SEIFERT, J.R.; MENGEL, D.B. \& POPE, P.E. Defining competition vectors in a temperate alley cropping system in the midwestern USA. 3. Competition for nitrogen and litter decomposition dynamics. Agrofor. Syst., 48:61-77, 2000.

KANG, B.T. Alley cropping - soil productivity and nutrient recycling. For. Ecol. Manag., 97:75-82, 1997.

KAYA, B. \& NAIR, P.K.R. Soil fertility and crop yields under imporvedfallow systems in Southern Mali. Agrofor. Syst., 52:111, 2001.

LOSS, A.; PEREIRA, M.G.; SCHULTZ, N.; ANJOS, L.H.C. \& SILVA, E.M.R. Atributos químicos e físicos de um Argissolo Vermelho-Amarelo em sistema integrado de produção agroecológica. Pesq. Agropec. Bras., 44:68-75, 2009.

MacDICKEN, K.G. \& VERGARA, N.T. Agroforestry: Classification and management. New York, John Wiley \& Sons, 1990. 382p.

MAIA, S.M.F.; XAVIER, F.A.S.; SENNA, O.T.; MENDONCA, E.S. \& ARAUJO, J.A. Organic carbon pools in a Luvisol under agroforestry and conventional farming systems in the semi-arid region of Ceará, Brazil. Agrofor. Syst., 71:127-138, 2007.

MAJUMDER, B.; MANDAL, B.; BANDYOPADHYAY, P.K.; GANGOPADHYAY, A.; MANI, P.K.; KUNDU, A.L. \& MAZUMDAR, D. Organic amendments influence soil organic carbon pools and rice-wheat productivity. Soil Sci. Soc. Am. J., 72:775-785, 2008.

MENDONÇA, E.S. \& STOTT, D.E. Characteristics and decomposition rates of pruning residues from a shaded coffee system in Southeastern Brazil. Agrofor. Syst., 57:117-125, 2003

MORAES, A.G.L.; LOSS, A.; PEREIRA, M.G.; BERNINI, T.A.; ANJOS, L.H.C.; SANTOS, L.L. \& WADT, P.G. Frações oxidáveis de carbono orgânico de horizontes superficiais em topossequência sob floresta no Acre. In: FERTBIO, 2008. Desafios para o uso do solo com eficiência e qualidade ambiental. Londrina, 2008. Anais. Londrina, 2008. CD-ROM.

MOURA, E.G. \& AGUIAR, A.C.F. Plantio direto na palha de leguminosas em aleias, uma alternativa agroecológica para a agricultura familiar do trópico úmido. In: CONGRESSO BRASILEIRO DE SISTEMAS DE PRODUÇÃO, 7., Fortaleza, 2007. Anais. Fortaleza, 2007.

NAIR, P.K.R. Nutrient cycling in tropical agroforestry systems: myths and science. In: BUCK, L.E.; LASSOIE, J.P. \& FERNANDES, E.C.M., eds. Agroforestry in sustainable agricultural aystems. Boca Raton, CRC Press, 1999. p.1-31.

RANGEL, O.J.P.; SILVA, C.A.; GUIMARÃES, P.T.G. \& GUILHERME, L.R.G. Frações oxidáveis do carbono orgânico de Latossolo cultivado com cafeeiro em diferentes espaçamentos de plantio. Ci. Agrotecnol., 32:429-437, 2008.

SALMI, G.P.; SALMI, A.P. \& ABBOUD, A.C.S. Dinâmica de decomposição e liberação de nutrientes de genótipos de guandu sob cultivo em aleias. Pesq. Agropec. Bras., 41:673678,2006

SHARROW, S.H. \& ISMAIL, S. Carbon and nitrogen storage in agroforests, tree plantations, and pastures in Western Oregon, USA. Agrofor.Syst., 60:123-130, 2004. 
STEVENSON, F.J. Humus chemistry: Genesis, composition, reactions. New York, J. Willey, 1994. 456p.

WALKLEY, A. \& BLACK, J.A. An examination of the Degtjareff method for determining soil organic matter and a proposed modification of the chromic titration method. Soil Sci., 37:29-38, 1934.
YEOMANS, J.C. \& BREMNER, J.M. A rapid and precise method for routine determination of organic carbon in soil. Comm. Soil Sci. Plant Anal., 19:1467-1476, 1988.

YOUNG, A. Agroforestry for soil management. New York: CAB International, 1997. 320p. 